\title{
Stratégies pour augmenter le nombre de donneurs d'organes
}

\author{
II existe un consensus sur le fait que des mesures sont nécessaires pour augmenter \\ le nombre de donneurs d'organes. Mais lesquelles? Promotion du don vivant, rému- \\ nération financière, modèle de réciprocité, dispositions légales? Chaque approche \\ recèle son lot de pour et de contre.
}

Julius Weiss, Marc Marthaler, Franz F. Immer

Fondation nationale Suisse pour le don et la transplantation d'organes
Correspondance:

PD Dr Franz F. Immer FMH chirurgie cardiaque et vasculaire thoracique Directeur Swisstransplant Laupenstrasse 37

CH-3008 Berne

Tél. 0313808133

Fax 0313808132

franz.immer@ swisstransplant.org

www.swisstransplant.org
L'offre d'organes pour la transplantation est trop faible depuis des années et la demande continue d'augmenter de manière soutenue - ce qui a des conséquences graves pour les personnes qui ont besoin d'un organe et se retrouvent sur une liste d'attente de plus en plus longue. Il existe donc un large consensus sur la nécessité urgente de prendre des mesures énergiques pour augmenter le nombre de donneurs [1-3]. Les opinions divergent d'avantage quant aux moyens qui permettraient le mieux d'atteindre cet objectif. Plusieurs approches sont en discussion - mais toutes les parties prenantes s'accordent à dire qu'il n'est plus temps de débattre, mais qu'il faut agir avec détermination.

En réalité, les cercles impliqués dans la transplantation d'organes ne sont pas restés inactifs jusqu'ici. Les mesures les plus diverses ont été et sont encore prises pour augmenter le nombre de donneurs: des campagnes d'information destinées à la population, une meilleure détection des donneurs dans les hôpitaux, la réintroduction du programme des donneurs à cœur non battant en Suisse, pour n'en citer que quelques-unes. Même si chacune de ces mesures contribue précieusement à améliorer la situation, on n'a malheureusement pas encore assisté jusqu'à présent à une véritable percée. Certes, on note depuis peu en Suisse une légère tendance à la hausse du nombre de donneurs; celle-ci démarre toutefois à un niveau relativement bas et ne peut donc compenser le sérieux manque d'organes.

La société se doit envers ses membres les plus faibles - les malades qui ont urgemment besoin d'un organe - de trouver au plus vite une solution à ce problème. Car différer la solution, cela revient dans le pire des cas à laisser mourir des personnes qui pourraient vivre s'il y avait davantage de donneurs. Tout le monde est donc invité à apporter sa contribution. Les solutions possibles sont connues - et chacun sait aussi que ne rien faire n'est pas une alternative. Afin d'aider chacun à décider, qu'il s'agisse de la décision personnelle pour ou contre le don d'organes, ou bien de la décision politique tout aussi importante qui consiste à opter pour des solutions face au problème du manque d'organes, cet article donne un aperçu des différentes actions possibles. Celles-ci incluent la promotion du don d'organes, l'indemnisation financière du don d'organes, le modèle de réciprocité, ainsi que la promotion du don d'organes via des dispositions législatives.

\section{Promotion du don d'organes}

\section{Idée fondamentale}

Le don de rein, de foie, d'intestin et de poumon est possible du vivant du donneur. La liste d'attente pour la plupart des organes est si longue qu'elle ne peut être réduite uniquement grâce au don d'organes de donneurs décédés, ce qui a de graves conséquences pour la qualité de vie des patients en liste d'attente et engendre des coûts considérables, qui seraient évités si davantage d'organes étaient mis à disposition. Si plus de personnes donnaient un organe de leur vivant, ces conséquences négatives du manque d'organes pourraient être atténuées, du moins en partie.

\section{Chacun sait que ne rien faire n'est pas une alternative}

\section{Pour}

Le don d'organe d'une personne vivante permet de raccourcir le délai d'attente pour l'attribution d'un organe et de prévoir de manière optimale le moment de la transplantation. Ceci signifie que les résultats après un don d'organe d'une personne vivante sont souvent considérablement meilleurs qu'après celui d'une personne décédée [4] En cas de transplantation précoce, comme c'est possible pour les dons de reins de personnes vivantes (la forme la plus courante de don du vivant du donneur), l'autre avantage est que la transplantation, comparée à la dialyse, est à la fois plus économique et associée à une amélioration significative de la qualité de vie. 


\section{Contre}

Certes, le don d'organes est basé sur le principe du libre choix (loi sur la transplantation, al. 3), mais il n'est pas exclu dans le cas du don d'organe en faveur de membres de la famille, que le donneur éprouve un certain sentiment d'obligation morale ou même qu'il soit conduit à faire ce don d'organe de son vivant par une pression implicite de la famille.

L'autre inconvénient est qu'un don d'organe du vivant du donneur comporte pour celui-ci un risque même minime. Par ailleurs, l'incertitude demeure quant à la conduite à adopter lorsqu'une personne qui a donné un organe a elle-même besoin d'une transplantation par la suite. Jusqu'ici, il n'est pas prévu de considérer, lors de l'attribution d'un organe, si une personne a déjà fait don d'un organe, comment cela semble justifié dans ce cas. En dehors du cercle familial, on peut en outre difficilement s'attendre à ce que beaucoup de personnes prennent le risque d'un tel don par altruisme alors qu'elles n'ont pas de lien personnel avec le bénéficiaire.

\section{Indemnisation financière du don d'organes}

Bien qu'en Suisse, la non-rétribution du don d'organes soit ancrée dans la loi fédérale régissant la transplantation d'organes, de tissus et de cellules (loi sur la transplantation d'organes, art. 6) et que le commerce d'organes soit interdit (art. 7), l'indemnisation financière du don d'organes doit cependant être discutée ici, car elle ne cesse d'être évoquée comme solution possible au manque d'organes.

\section{Idée fondamentale}

Là où une demande existe, un marché avec une offre correspondante se développe. Étant donné qu'il y a trop peu de donneurs d'organes qui mettent gratuitement leurs organes à disposition, l'indemnisation financière peut constituer une motivation.

Pour

Le monde dans lequel nous vivons n'est pas parfait; car dans un monde parfait, les donneurs d'organes seraient en nombre suffisant. Or il existe un manque d'organes qui a pour conséquence le commerce d'organes dans certains pays [5]. Ceci est certes illégal dans presque tous les pays, mais cela ne veut pas dire pour autant, comme il en va avec d'autres marchés illégaux, que le commerce d'organes ne se pratique pas. L'une des conséquences est que ce ne sont ni ceux qui acceptent plus ou moins de leur plein gré de donner leurs organes, ni les bénéficiaires de ces organes qui en profitent en premier lieu, mais des intermédiaires, qui organisent et gèrent le commerce illégal. Une règlementation nationale ou internationale du commerce d'organes semble donc être la seule solution pour minimiser les conséquences négatives qu'entraîne un marché non contrôlé, aussi bien pour les offrants que pour les bénéficiaires. [6].

\section{Contre}

Certains craignent que la rémunération du don d'organes n'affaiblisse la motivation des personnes de mettre gratuitement leurs organes à disposition, et que par conséquent cela ne débouche pas globalement sur une hausse du nombre de donneurs [7]. En outre, on peut supposer que ce sont surtout des personnes défavorisées socialement qui proposeraient leurs organes contre rémunération. Ceci serait perçu comme l'exploitation d'une situation de dénuement, ce qui pose un grand problème sur le plan moral et ne constitue donc pas une solution envisageable [8].

\section{Modèle de réciprocité}

\section{Idée fondamentale}

Il ne peut y avoir de transplantations d'organes que s'il y a des donneurs d'organes - de même qu'on ne peut offrir un cadeau que s'il y a quelque chose à offrir. Cela signifie que toute personne n'excluant pas la possibilité de se voir transplanter un organe en cas de nécessité devrait par conséquent accepter aussi d'être donneur d'organe [9]. On a du mal à comprendre pourquoi des gens qui refusent de donner leurs organes devraient avoir le droit de bénéficier d'un don d'organe; car si tout le monde se comportait ainsi, il n'y aurait plus du tout d'organes disponibles pour une transplantation.

\section{Pour}

Le risque au cours d'une vie de devoir un jour recourir à une greffe d'organe est sept fois supérieur à la probabilité de devenir soi-même donneur. Vu sous cet angle, donner son accord pour un don d'organe présente certaines similitudes avec le fait de souscrire une assurance. Il n'est donc pas logique que des personnes qui ne sont pas prêtes à apporter une modeste contribution puissent tout de même profiter pleinement des avantages de la transplantation d'organes en cas de besoin [7]. D'autre part, cette possibilité d'adopter une conduite opportuniste peut faire paraître le système injuste et dissuader les personnes de consentir au don d'organes.

\section{Contre}

Accorder la préférence aux donneurs déclarés par rapport aux non-donneurs contredit le principe selon lequel personne ne doit être l'objet de discrimination s'agissant de l'attribution d'organes (loi sur la transplantation, art. 17). Cela signifierait en outre que des critères non médicaux sont pris en compte lors de l'attribution, ce qui est exclu jusqu'ici à juste titre.

L'incertitude demeure quant à la manière de mettre en œuvre un tel modèle de réciprocité, de sorte que la réciprocité puisse être effectivement prise en compte comme un critère juste lors de l'attribution d'organes: comment exclure la possibilité que des personnes ne remplissent une carte de donneur que parce 


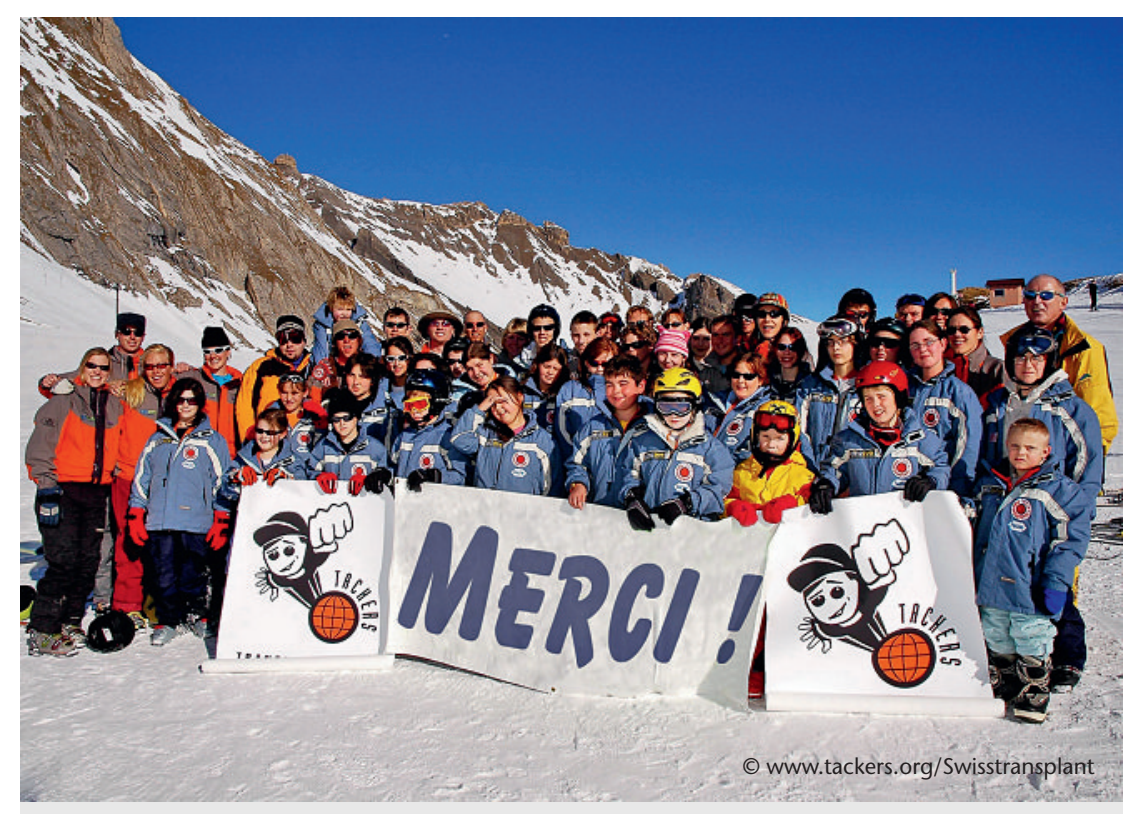

Le don d'organes - une nouvelle vie offerte: les participants du camp d'hiver pour des enfants transplantés «TACKERS» (Transplant Adventure Camps for Kids).

qu'elles savent qu'elles ont elles-mêmes besoin d'une transplantation (contournant ainsi l'idée de réciprocité)?

\section{Promotion du don d'organes grâce à des dispositions législatives}

\section{Idée fondamentale}

Il est dans l'intérêt des personnes qui ont besoin d'une transplantation qu'il y ait le plus de donneurs possibles. De plus, comparé à d'autres formes de thérapie, une transplantation s'avère généralement plus économique sur le long terme, de sorte que promouvoir le don d'organes va à cet égard aussi dans le sens de la communauté. Il semble donc judicieux que le législateur promulgue des dispositions visant à ce que le plus de personnes possibles acceptent de faire un don d'organe.

\section{La solution de l'opposition contre la solution du consentement}

Une option consisterait à adopter dans la loi sur la transplantation la solution de l'opposition au lieu de la solution du consentement actuellement en vigueur - à savoir qu'un prélèvement d'organe sur une personne décédée n'est possible que si celle-ci (ou ses proches) a donné au préalable son consentement explicite. La solution de l'opposition prévoit que toute personne n'ayant pas stipulé explicitement qu'aucun de ses organes ne doit être prélevé après sa mort soit considérée comme un donneur d'organes potentiel.

\section{Pour}

Dans de nombreux pays, une majorité de la population, lors qu'elle est interrogée dans des sondages, s'exprime en faveur du don d'organes [10]. On peut donc en déduire que le don d'organes représente une attitude approuvée socialement. Le législateur peut donc en tirer la conclusion que le consentement au don d'organes est considéré comme donné et que la loi devrait stipuler que les personnes qui refusent de donner leurs organes sont tenues de le mentionner explicitement, et non celles qui l'acceptent.

\section{Contre}

Il n'est pas certain que la solution de l'opposition augmenterait de manière importante le nombre de donneurs. Ce qui est sûr, c'est qu'il serait naîf de croire que la simple introduction de la solution de l'opposition se traduirait automatiquement par une augmentation considérable [11]. Il reste en outre à clarifier quels effets ce règlement aurait sur les enfants et les personnes incapables de discernement.

\section{Rendre le don d'organes post-mortem obligatoire}

Dans un contexte comme celui du don d'organes, où le libre choix et l'altruisme revêtent une importance majeure, il semble à première vue étonnant d'évoquer la possibilité de l'obligation. Mais si l'on considère le don d'organes comme une forme de secours d'urgence - à savoir sauver indirectement la vie à quelqu'un -, alors l'idée que l'on pourrait y être contraint par la loi (de même qu'il existe une obligation de venir en aide aux personnes exposées à un danger de mort immédiat) n'apparaît plus si aberrante [12].

\section{Pour}

Il existe différents devoirs civiques qui limitent la liberté de l'individu; outre le secours d'urgence, par exemple le service militaire ou l'obligation de payer ses impôts. Si l'on tient compte du fait qu'une transplantation d'organe peut sauver la vie d'un patient, alors, si l'on procède à une pesée des intérêts, il semble qu'une restriction de la liberté de choix dans le cas du don d'organe post-mortem se justifie. Le critère de l'acceptabilité, qui limite la portée du secours d'urgence, contredirait l'obligation du don d'organes du vivant de la personne (du fait que cela présente un certain risque pour la santé du donneur). Dans le cas du don post-mortem, le donneur n'encourt en revanche pas de risques qui pourraient être mis en balance avec le profit qu'en retire le patient bénéficiant d'une transplantation [13].

\section{Contre}

Dans le cas d'un refus du don d'organes, la volonté exprimée de son vivant par la personne décédée (ou la volonté de ses proches) doit être considérée comme plus importante que l'intérêt de ceux qui ont besoin d'un organe [14]. Rendre le don d'organes post-mortem obligatoire peut entrer en contradiction avec des convictions religieuses ou avec une vision du monde et ainsi empiéter de manière excessive sur la liberté personnelle, de sorte que par principe, une telle solution ne peut être acceptée. 


\section{Bilan}

Les stratégies présentées ici en vue d'augmenter le nombre des donneurs d'organes ont toutes - comme il fallait s'y attendre - des avantages et des inconvénients. Il n'y a donc pas de stratégie qui résoudrait le problème du manque d'organes sans qu'il faille craindre certaines conséquences regrettables. Cepen-

\section{«Pour améliorer la situation, quels inconvénients pouvons-nous accepter?»}

dant, cela ne peut justifier que l'on ne prenne aucune décision. En effet, ne pas trancher empêche que l'on puisse aider des personnes qui, si elles ne bénéficient pas d'une transplantation, voient leur qualité de vie extrêmement diminuée, ou même risquent de décéder parce qu'elles doivent attendre trop longtemps en raison du manque d'organes disponibles. La question à laquelle nous devons répondre est la suivante: «Pour améliorer la situation, quels inconvénients pouvons-nous accepter?» Et une fois la réponse trouvée, il faut la mettre en œuvre.

\section{Références}

1 Nett PC, Stüssi G, Weber M, Seebach JD. Transplantationsmedizin: Strategien gegen den Organmangel. Schweiz Med Forum. 2003;24(11):559-68.

2 Abouna GM. Organ shortage crisis: problems and possible solutions. Transplant Proc. 2008;40:34-8.

3 Flückiger A (Hrsg.). Emouvoir et persuader pour promouvoir le don d'organes? - L'efficacité entre éthique et droit. Genève: Schulthess; 2010.
4 Thiel GT. Nieren-Lebendspende im Grenzgebiet von Klinik, Ethik und Recht. Kodex für den Umgang mit Lebendspendern. In: Breyer F, Engelhard M (Hrsg.). Anreize zur Organspende. Graue Reihe, Nr. 39. Europäische Akademie zur Erforschung von Folgen wissenschaftlich-technischer Entwicklungen Bad Neuenahr-Ahrweiler GmbH; 2006; S. 143-170.

5 Steering Committee of the Istanbul Summit. Organ trafficking and transplant tourism and commercialism: the Declaration of Istanbul. Lancet. 2008;372:5-6.

6 Friedman EA, Friedman AL. Payment for donor kidneys: Pros and Cons. Kidney Int. 2006;69:960-2.

7 Illies C, Weber F. Organ trade versus reciprocity model. An ethical evaluation. Dtsch Med Wochenschr. 2004;129:271-5.

8 Budiani-Saberi DA, Delmonico FL. Organ trafficking and transplant tourism: a commentary on the global realities. Am J Transplant. 2008;8:925-9.

9 Lavee J, Ashkenazi T, Gurman G, Steinberg D. A new law for allocation of donor organs in Israel. Lancet. 2010;375:1131-3.

10 European Commission. Special Eurobarometer 272D «Europeans and organ donation». 2007.

11 Rithalia A, McDaid C, Suekarran S, Myers L, Sowden A. Impact of presumed consent for organ donation on donation rates: a systematic review. BMJ. 2009;338:a3162.

12 Wille S. Sozialpflicht zur Organspende? In: Breyer F, Engelhard M (Hrsg.). Anreize zur Organspende. Graue Reihe Nr. 39. Europäische Akademie zur Erforschung von Folgen wissenschaftlich technischer Entwicklungen Bad NeuenahrAhrweiler GmbH; 2006; S. 7-26.

13 Spital A, Taylor JS. Routine recovery of cadaveric organs for transplantation: consistent, fair, and life-saving. Clin J Am Soc Nephrol. 2007;2:300-3.

14 Hamer CL, Rivlin MM. A stronger policy of organ retrieval from cadaveric donors: some ethical considerations. J Med Ethics. 2003;29:196-200. 